MS44-03

\section{The use of Principal Component Analysis for fast and efficient kinetic analysis of combined in situ $X$-ray diffraction and spectroscopic data}

Marco Milanesio ${ }^{1}$, Rocco Caliandro ${ }^{2}$, Luca Palin ${ }^{3}$, Eleonora

Conterosito $^{3}$

1. Dipartimento di Scienze e Innovazione Tecnologica, Alessandria, Italy

2. IC-CNR, Bari, Italy

3. Università del Piemonte Orientale, Alessandria, Italy

email: marco.milanesio@uniupo.it

Combining in situ X-ray powder diffraction (XRPD) and spectroscopic data opened new avenues in the studies of solid-state reactivity and kinetics. The obtained dataset is complex and large and efficient methods of analysis are not available. Recently Principal Component Analysis (PCA) resulted suitable for the fast kinetic analysis of in situ XRPD data [1-3]. Real and simulated in situ XRPD data were analysed to unravel the potentialities and limitation of the approach. In the simulations, the changing parameters (weight fractions, lattice parameters and crystallite sizes of reactant and products) were varied, at first separately and then together to understand the capability of PCA to untangle the kinetic information in cases of different complexity. Finally, experimental isothermal and non-isothermal real case data were analysed by PCA to explore real world applications. Then real experimental data on a complex case, the Maya Blue pigment formation are presented. It is generally accepted that $\mathrm{MB}$ can be obtained by thermal treatment of a mixture of naturally occurring minerals as inorganic framework (typically containing palygorskite), and an indigo dye source. To unravel the features of the MB formation process in situ XRD/PDF data were collected, on several framework-dye couples, while in situ FORS, TGA and dye leaching experiments were carried out on preheated palygorskite/ indigo mixtures. Statistical PCA-based methods were exploited to efficiently and jointly extract the chemical information from the more than 1500 experimental patterns. MB formation starts below $110^{\circ} \mathrm{C}$ (from PDF analyses), reaching maximum reaction speed at $150^{\circ} \mathrm{C}$ (shown by XRD/ TGA). Above $175^{\circ} \mathrm{C}$, peculiar reactions occur with color changes, a stronger sequestration of indigo into the channels and changes in the affinity for water (proved by leaching and FORS experiments). The cooling to RT resulted important in defining the penetration and degree of sequestration of indigo into the palygorskite channels. The different colors, hues and stability in ancient and modern MB can be now explained by the thermal history of each sample. PCA performed rather well in non-isothermal in situ studies, and it can be used both for on-line data monitoring to check experiment data quality, and to efficiently unravel the reaction coordinate to successfully carry out kinetic analysis in in situ XRPD/PDF and spectroscopic data.
References:

[1] L. Palin, R. Caliandro, D. Viterbo, M. Milanesio, 2015, Phys. Chem. Chem. Phys., 17, 17480-93.

[2] L. Palin, E. Conterosito, R. Caliandro, E. Boccaleri, G. Croce, S. Kumar, W. van Beek, M. Milanesio, 2016, CrystEngComm, 18, 5930-5939.

[3] P. Guccione, L. Palin, M. Milanesio, B. D. Belviso, R. Caliandro, 2018, Phys. Chem. Chem. Phys., 20, 2175-2187.

Keywords: In situ X-ray Diffraction, Kinetic analysis, Principal Component Analysis 